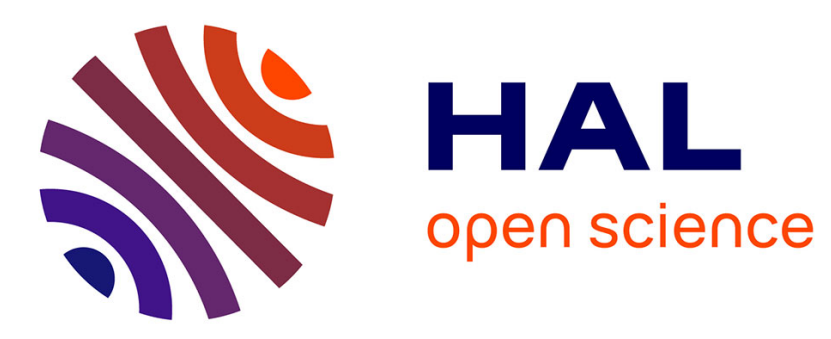

\title{
Le champ de coupure des magnétrons
}

\author{
J.-M. Rocard
}

\section{To cite this version:}

J.-M. Rocard. Le champ de coupure des magnétrons. Revue de Physique Appliquée, 1967, 2 (4), pp.275-282. 10.1051/rphysap:0196700204027500 . jpa-00242805

\section{HAL Id: jpa-00242805 https://hal.science/jpa-00242805}

Submitted on 1 Jan 1967

HAL is a multi-disciplinary open access archive for the deposit and dissemination of scientific research documents, whether they are published or not. The documents may come from teaching and research institutions in France or abroad, or from public or private research centers.
L'archive ouverte pluridisciplinaire HAL, est destinée au dépôt et à la diffusion de documents scientifiques de niveau recherche, publiés ou non, émanant des établissements d'enseignement et de recherche français ou étrangers, des laboratoires publics ou privés. 


\title{
LE GHAMP DE GOUPURE DES MAGNÉTRONS
}

\author{
Par J.-M. ROGARD, \\ Laboratoire de Physique des Milieux Ionisés, Faculté des Sciences, Toulouse.
}

\begin{abstract}
Résumé. - On rappelle tout d'abord que la relation donnant le champ de coupure d'un magnétron cylindrique à cathode filiforme $\left(B_{\mathrm{c}}=\sqrt{\frac{8 m}{e}} \frac{V^{1 / 2}}{R}\right)$ n'est réellement valable que lorsque les effets de charges d'espace peuvent être négligés. Lorsque ces derniers deviennent importants, dans la relation de conservation de l'énergie appliquée à un électron individuel, il faut tenir compte des interactions électron-électron dues aux charges d'espace du nuage anodique, quand on désire déduire la " condition de coupure ". Il en résulte un phénomène de déplacement vers les champs faibles du champ de coupure effectif, quand le niveau d'émission électronique augmente. Ce phénomène apparait très nettement sur les graphiques $I_{\mathrm{a}} / I_{\mathrm{a}}(\mathrm{o})=f(B)$ où $I_{\mathrm{a}}(\mathrm{o})$ représente le courant anodique recueilli par l'anode quand le champ magnétique est nul et que le potentiel d'anode $V$ est fixé. Des résultats expérimentaux sont donnés qui montrent l'importance de cet effet : une diminution relative de $12 \%$ sur la valeur de $B_{\mathrm{c}}$ a été mesurée quand $I_{\mathrm{a}}(\mathrm{o})$ varie de $10^{-7}$ à $10^{-3}$ ampère.
\end{abstract}

Abstract. - It is pointed out first that the cut-off relation $\left(B_{\mathrm{c}}=\sqrt{\frac{8 m}{e}} \frac{V^{1 / 2}}{R}\right)$ for a cylindrical magnetron is valid only when space charge effects can be neglected. When this is not the case, the energy conservation principle, used in order to get the "cut-off" condition, requires that the interaction between an individual electron and the electrons of the anode cloud should be taken into account. A displacement of the cut-off field towards low values when the electronic emission level increases, should be and is observed. Our experimental results, as well as previous works (Hull, Lafferty), confirm this conclusion : a relative displacement $\Delta B_{\mathrm{c}} / B_{\mathrm{c}}$ of the order of $12 \%$ has been measured when the electronic emission level varies from $10^{-7}$ to $10^{-3}$ ampere.

Introduction. - Le magnétron de Hull est constitué de deux conducteurs cylindriques coaxiaux, placés dans un champ magnétique longitudinal : le cylindre intérieur sert d'émetteur d'électrons, le cylindre extérieur est le collecteur lorsqu'une différence de potentiel convenable est appliquée entre les deux électrodes. Si le champ magnétique $\mathrm{B}$ uniforme, parallèle à l'axe, est suffisamment intense, les électrons n'atteignent pas le collecteur; on dit qu'il y a coupure. De nombreux auteurs, après Hull [1], ont étudié, tant théoriquement (Brillouin [2], Allis [3], Page et Adams [4], Delcroix [5], Coste [6]) qu'expérimentalement (Hull [1], Reverdin [7], Delcroix [5]) les problèmes posés par les distributions de champ électrique et de charges d'espaces d'un magnétron en régime de coupure. Dans ces études, les effets des collisions électroniques avec les molécules du gaz résiduel sont négligeables; or, ce n'est pas toujours le cas. Une publication de Redhead [8] fait sans doute état pour la première fois de la possibilité d'utiliser à la mesure des très basses pressions $\left(10^{-3}\right.$ à $10^{-12}$ torr $)$ une diode cylindrique à cathode froide placée dans un champ magnétique longitudinal.
Une étude détaillée d'un type de jauge analogue (à filament chaud) est ensuite effectuée par J. M. Lafferty [9] dans le but de mettre au point un appareil de mesure convenable, délivrant un courant proportionnel à la pression dans une assez large gamme. Enfin Kaufman [10] et d'autres après lui ont utilisé le principe du magnétron de Hull pour construire des sources d'ions à forte intensité, nécessaires à un fonctionnement efficace des moteurs ioniques.

En dehors de ces applications techniques, il est certain que l'étude de ce genre de tube peut fournir des renseignements sur le fonctionnement du magnétron lui-même, dont les propriétés ne sont pas totalement connues, comme l'attestent les nombreux articles déjà cités. En particulier, nous avons voulu étudier un phénomène de déplacement du champ de coupure en fonction du niveau d'émission de la cathode.

Pour des émissions très faibles, quand les charges d'espace sont négligeables, le champ magnétique nécessaire à une coupure efficace du courant anodique est supérieur à celui qui correspond à des émissions 
intenses, toutes les autres conditions étant identiques (potentiel et rayon d'anode).

L'objet de ce rapport est de mettre en évidence ce phénomène de déplacement du champ de coupure. Le calcul des trajectoires et des vitesses des électrons dans des magnétrons plan ou cylindrique (effets des charges d'espace négligés) montre que la distribution des charges dans l'espace interélectrode est affectée par la présence du champ magnétique; quand il n'y a pas de champ magnétique, la concentration d'électrons diminue très rapidement à mesure que l'on s'éloigne de la cathode; par contre, lorsque le champ magnétique est de l'ordre de $B_{\mathrm{e}}$ (champ de coupure), les densités volumiques d'électrons se répartissent non seulement près de la cathode, mais aussi près de l'anode. Les charges d'espace anodique (lorsque le niveau d'émission cathodique est tel que l'on ne peut plus négliger les effets de charge d'espace) peuvent empêcher un électron, quittant la région cathodique, d'acquérir toute l'énergie potentielle $\mathrm{eV}$ qu'il possède en atteignant l'anode si les effets de charge d'espace sont négligeables (émission du filament faible).

Ce déplacement du champ de coupure est observable sur des caractéristiques expérimentales de magnétron publiées par certains auteurs (Hull-Lafferty), mais ne pouvait être attribué aux effets de charge d'espace parce que, $1^{\circ}$, dans le cas de Hull, les caractéristiques n'avaient pas pu être relevées pour les très faibles émissions (limitation due à la pression résiduelle trop élevée) et $2^{\circ}$, dans le cas de Lafferty, la forme en " épingle à cheveux » du filament introduisait des conditions géométriques compliquées.

Les caractéristiques expérimentales que nous présentons ont été obtenues avec une diode magnétron cylindrique, placée dans un système à ultra-vide pouvant atteindre $10^{-9}$ torr. Les très basses pressions sont, en effet, nécessaires pour qu'il soit possible de négliger les effets dus aux collisions électroniques avec les molécules neutres du gaz résiduel.

Le champ de coupure. - RAPPEL DES RÉsultats CLASSIQUES (charges d'espace négligeables). - La figure 1 représente, en projection sur un plan perpendiculaire à l'axe, un magnétron de Hull. La trajectoire d'un électron, quittant le filament $F$, est courbée par le champ d'induction magnétique $\mathbf{B}$ uniforme, parallèle à $\mathrm{F}$. Si le champ magnétique est assez fort, il peut empêcher les électrons émis d'atteindre l'anode.

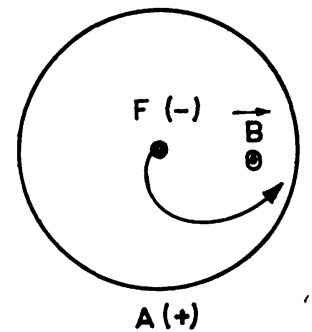

FIG. 1. - Principe de fonctionnement du magnétron.
Comme tous les électrons situés à une distance $r \mathrm{du}$ filament ont la même vitesse (les vitesses initiales peuvent être négligées lorsque le champ électrique radial $E$ est suffisamment intense), il existe un champ critique $B_{\mathrm{o}}$ au-dessus duquel le courant anodique est interrompu et la caractéristique classique du tube est alors représentée par la courbe discontinue de la

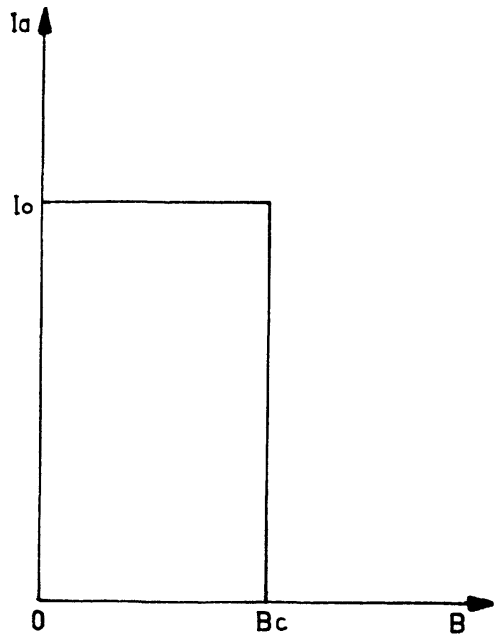

FIG. 2. - Caractéristiques classiques du magnétron.

figure 2 (courant anodique en fonction du champ magnétique $B$ ).

L'application du principe fondamental de la dynamique à un électron se déplaçant entre le filament et l'anode, ou du principe de conservation de l'énergie à cet électron, conduit à la relation (1) qui donne l'expression du champ de coupure en fonction de la différence de potentiel appliquée entre les électrodes $(V)$ et le rayon $R$ du magnétron :

$$
B_{\mathrm{c}}=\sqrt{\frac{8 m}{e}} \frac{V^{1 / 2}}{R} .
$$

Cette relation ne fait intervenir ni la répartition du potentiel ni celle des charges d'espace entre cathode et anode. Le champ de coupure devrait donc être le même quel que soit le régime d'émission du filament (limitation par les effets de charges d'espace ou limitation par la température dans le cas des très faibles émissions). Plusieurs questions se posent donc immédiatement : quelle est la définition du champ de coupure expérimental, et ce champ de coupure expérimental une fois défini vérifie-t-il les propriétés qui viennent d'être énoncées? Si l'on se réfère aux caractéristiques expérimentales $I_{\mathrm{a}}=f(B)$ données par Hull, on constate que la valeur théorique de $B_{\mathrm{c}}$, calculée à partir de la relation (1), correspond à la coupure presque totale du courant anodique et non pas au début de la coupure effective (fin du plateau $I_{\mathrm{a}}=I_{\mathrm{a}}(\mathrm{o})$ ) comme on pourrait s'y attendre d'après 
la relation exprimant le principe de conservation de l'énergie :

$$
\left(\frac{\mathrm{d} r}{\mathrm{~d} t}\right)^{2}+\frac{r^{2} e^{2} B^{2}}{4 m^{2}}=\frac{2 e V}{m}
$$

en effet, pour des valeurs de $B$ légèrement inférieures à $B_{\mathrm{e}}$, la vitesse radiale $\frac{\mathrm{d} r}{\mathrm{~d} t}$ des électrons n'est pas nulle et, par conséquent, tous les électrons devraient être recueillis par l'anode, donc $I_{\mathrm{a}}\left(B \leqslant B_{\mathrm{o}}\right)=I_{\mathrm{a}}(\mathrm{o})$.

Pour éviter cette difficulté d'interprétation, les résultats sont présentés dans ce rapport sous la forme $\frac{I_{\mathrm{a}}}{I_{\mathrm{a}}(\mathrm{o})}=f(B)$ où $I_{\mathrm{a}}(\mathrm{o})$ est le courant recueilli par l'anode lorsque le champ magnétique $B$ est nul. De cette façon, pour un magnétron donné (rayon $R$ et potentiel d'anode $V$ ), il est possible de comparer les caractéristiques obtenues sous de nombreux régimes d'émission électroniques et de confirmer l'existence d'un phénomène de déplacement du champ de coupure.

Ce phénomène de déplacement du champ de coupure étant attribué aux effets de charges d'espace anodiques, comme nous l'expliquerons dans la suite, il semble important de rappeler brièvement les principaux résultats déjà connus sur la répartition des densités volumiques d'électrons dans l'espace interélectrode d'un magnétron.

RÉPARTITION DES DENSITÉS VOLUMIQUES D'ÉLEGTRONS (MAGNÉTRONS PLAN ET GYLINDRIQUE). - Lorsque les effets de charge d'espace peuvent être négligés, la relation donnant le champ de coupure $B_{\mathrm{c}}$ peut être déduite du calcul de la trajectoire d'un électron. Celle-ci est cycloïdale dans le cas des magnétrons plans; elle est définie par l'équation polaire suivante :

$$
r=\left[\frac{k}{B} \sin \frac{2}{3} \theta\right]^{3 / 2}
$$

dans le cas des magnétrons cylindriques à cathodes filiformes ( $k$ dépend de $e, m, R$ et $V$ ) [1].

La densité de courant anodique dans une diode plane ordinaire $(B=0)$ est égale à :

$$
j_{0}=n_{z} e v_{z}
$$

avec

$$
v_{z}=\sqrt{\frac{2 e V_{z}}{m}}
$$

où $n_{z}, v_{z}$ et $V_{z}$ représentent respectivement la concentration, la vitesse des électrons et le potentiel à la distance $z$ de la cathode. Pour une différence de potentiel $V$ entre les électrodes, et à une température donnée de la cathode, la densité volumique des électrons décroît donc de façon continue et atteint sa valeur minimum au voisinage de l'anode; la courbe en trait plein de la figure 3 représente la variation

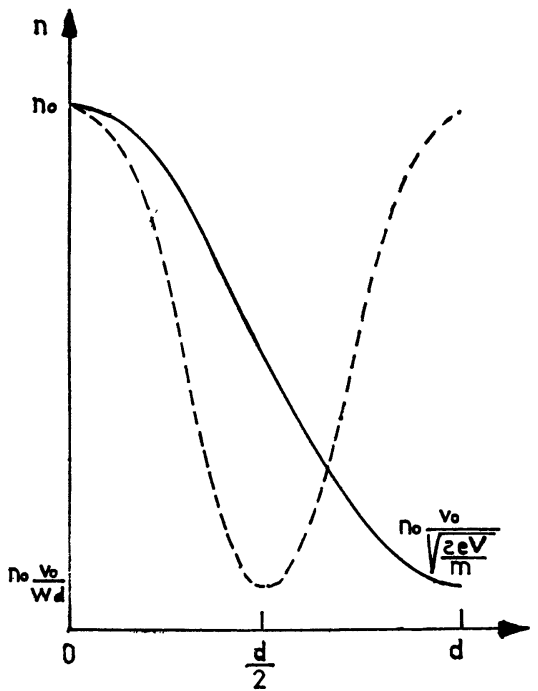

Fig. 3. - Répartition des concentrations d'électrons dans une diode plane avec et sans champ magnétique $\left(B \simeq B_{\mathrm{c}}\right)$ (effets des charges d'espace négligeables).

de $n_{z}$ en fonction de $z$ dans le cas où les effets des charges d'espace sont négligés. Quand la diode plane est soumise à un champ magnétique transversal $\left(B \leqslant B_{\mathrm{c}}\right)$, la densité de courant anodique est toujours donnée par la relation $j_{0}=n_{z} e v_{z}$, mais $v_{z}$ devient :

$$
v_{z}=\frac{\mathrm{d} z}{\mathrm{~d} t}=\frac{E}{B} \sin \omega_{\mathrm{b}} t+v_{\mathrm{o}} \cos \omega_{\mathrm{b}} t
$$

où $\omega_{\mathrm{b}}=\frac{e B}{m}$ et $v_{\mathrm{o}}$ est la vitesse initiale de l'électron.

La courbe en pointillé sur la figure 3 représente $n=f(z)$ quand le champ magnétique transversal est égal au champ de coupure $B_{\mathrm{o}}\left(B_{\mathrm{o}}=\sqrt{\frac{2 m}{e}} \cdot \frac{V^{1 / 2}}{d}\right)$ pour un magnétron plan; cette fonction présente un

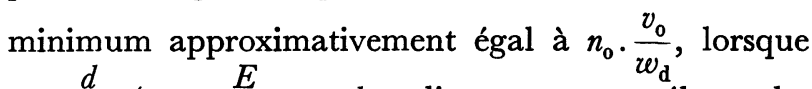
$z=\frac{d}{2} \quad\left(w_{\mathrm{d}}=\frac{E}{B}, \quad\right.$ et $d=$ distance entre électrodes planes).

Les deux courbes de répartition des densités électroniques de la figure 3 (avec et sans champ magnétique) font bien ressortir le rôle important du champ magnétique. Lorsque le champ magnétique est de l'ordre du champ de coupure, un nuage d'électrons s'accumule au voisinage de l'anode, ce nuage se déplaçant parallèlement à l'anode avec la vitesse de dérive $w_{\mathrm{d}}$.

La répartition des densités volurniques dans un magnétron cylindrique s'obtient soit à partir de la relation donnant la densité de courant comme précédemment, soit à partir de la relation donnant le courant anodique par unité de longueur du filament :

$$
i_{\mathrm{o}}=n_{\mathrm{r}} e v_{\mathrm{r}} 2 \pi r
$$




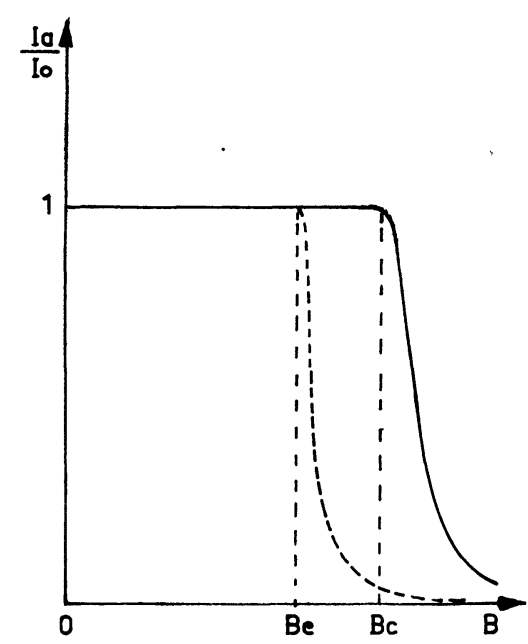

FIG. 4. - Caractéristiques théoriques de magnétrons (avec ou sans charges d'espace).

où $n_{\mathrm{r}}$ et $v_{\mathrm{r}}$ sont concentration et vitesse radiale des électrons à la distance $r$ du filament. La fonction $n_{\mathrm{r}}=f(r)$ présente un minimum pour $r=\left(\frac{1}{3}\right)^{3 / 4} R$ correspondant au maximum de $v_{\mathrm{r}}$ lorsque le champ magnétique longitudinal est égal au champ de coupure. Dans ces conditions ( $B \simeq B_{\mathrm{c}}$ ), un nuage d'électrons s'accumule au voisinage de l'anode cylindrique, ce nuage étant animé d'une vitesse angulaire constante $: \frac{\mathrm{d} \theta}{\mathrm{d} t}=\frac{\omega_{\mathrm{b}}}{2}$.

Tant que le niveau d'émission de la cathode (du magnétron plan ou cylindrique) reste peu élevé, les effets de charge d'espace de ce nuage peuvent être négligés; en d'autres termes, un électron quittant la cathode, sur son trajet vers l'anode, ne sera pas affecté par la prèsence de ce nuage anodique.

EFFETS DES GHARges D'ESPAGE ANODIQUES. - Lorsque l'émission de la cathode augmente suffisamment poúr que le courant anodique soit limité par les effets de charges d'espace $(B=0)$, aux deux relations exprimant la conservation de l'énergie et le théorème du moment cinétique, il faut ajouter l'équation de Poisson et celle qui exprime la continuité du courant émis par unité de longueur du filament.

Le système d'équations, pour un magnétron cylindrique à cathode filiforme, est donc :

$$
\left\{\begin{array}{l}
\left(\frac{\mathrm{d} r}{\mathrm{~d} t}\right)^{2}+r^{2}\left(\frac{\mathrm{d} \theta}{\mathrm{d} t}\right)^{2}=\frac{2 e}{m} V_{\mathrm{r}} \\
\frac{\mathrm{d}}{\mathrm{d} t}\left(r^{2} \frac{\mathrm{d} \theta}{\mathrm{d} t}\right)=\frac{e B}{m} r \frac{\mathrm{d} r}{\mathrm{~d} t} \\
\frac{1}{r} \frac{\mathrm{d}}{\mathrm{d} r}\left(r \frac{\mathrm{d} V_{\mathrm{r}}}{\mathrm{d} r}\right)=\frac{n_{\mathrm{r}} e}{\varepsilon_{\mathrm{o}}} \\
2 \pi r n_{\mathrm{r}} e \frac{\mathrm{d} r}{\mathrm{~d} t}=i_{0} .
\end{array}\right.
$$

Le régime de Brillouin [2] correspond à $\frac{\mathrm{d} r}{\mathrm{~d} t}=0$ pour toutes les valeurs de $r$ : les trajectoires des électrons sont des cercles.

Delcroix [5] a étudié en détail ce système d'équations quand le magnétron est en régime bloqué, en tenant compte des effets de charges d'espace : il en a déduit les conditions d'existence de régimes de Brillouin et de régimes bidromiques (l'électron après avoir quitté la cathode y revient) pour des magnétrons de formes géométriques diverses.

Quand les effets des charges d'espace ne peuvent plus être négligés, la répartition des densités volumiques d'électrons présente aussi un minimum entre la cathode et la surface de coupure lorsque la diode est soumise à un champ magnétique [5]; ce résultat a été vérifié expérimentalement (Reverdin [7]). Comme la condition de coupure est tirée de la première relation (système 2) exprimant la conservation de l'énergie, il est important de l'étudier en détail; cette relation est valable, qu'il y ait ou non un champ magnétique longitudinal pour $r<R$. Ainsi, quand un électron a réussi à s'échapper du nuage cathodique de charges d'espace, il atteint l'anode, son énergie potentielle $\mathrm{eV}$ étant égale à son énergie cinétique

$\frac{1}{2} m v^{2}$, si $B=0$ (pas de charges d'espace anodiques); par contre, lorsque le champ magnétique est de l'ordre du champ de coupure (légèrement inférieur), le même électron ayant quitté le nuage cathodique avec une vitesse initiale négligeable peut, sur son trajet vers l'anode, avoir des interactions avec les électrons du nuage anodique et, par conséquent, atteint l'anode avec une énergie potentielle inférieure à $\mathrm{eV}$ (donc $I_{\mathrm{a}}$ décroît). En d'autres termes, bien que le potentiel d'anode soit $V$ (potentiel de la cathode $=0$ ), quand on applique le théorème de conservation de l'énergie à un électron individuel entre l'instant où il a quitté le nuage d'électrons cathodique et celui où il atteint l'anode, on est obligé de tenir compte des effets de charges d'espace anodiques qui deviennent importants quand $B$ est voisin de $B_{c}$. Ce nuage électronique anodique animé d'une vitesse angulaire constante $\mathrm{d} \theta / \mathrm{d} t=\omega_{\mathrm{b}} / 2$ dans le cas du magnétron cylindrique ou d'une vitesse de dérive $w_{\mathrm{d}}$ dans le cas du magnétron plan constitue une montagne de potentiel à gravir par un électron individuel avant qu'il ne soit recueilli par l'anode même si les électrons qui constituent ce nuage sont constamment remplacés par de nouveaux électrons en provenance de la cathode.

Une relation empirique, tenant compte de cet effet, peut s'écrire de la façon suivante :

$$
\frac{1}{2} m v^{2}=e V-e \varphi
$$

où $v$ est la vitesse d'un électron quand il atteint l'anode, $V$ le potentiel d'anode et $\varphi$ le potentiel de charges d'espace anodique qui sera d'autant plus important 
que les concentrations d'électrons dans le nuage anodique le seront.

Ce potentiel $\varphi$ dépend donc non seulement du niveau d'émission cathodique $\left(n_{\mathrm{e}}\right)$, mais aussi de la valeur du champ magnétique $: \varphi=0$ si $B=0$, $\varphi$ est maximum pour $B=B_{\mathrm{c}}$. Dès que $B>B_{\mathrm{c}}$, ce terme n'a plus d'intérêt, la relation de la conservation d'énergie redevient :

$$
\frac{1}{2} m v^{2}=e V_{\mathrm{r}} \quad \text { avec } \quad r<R .
$$

A partir de la relation (3), on peut obtenir les valeurs des champs de coupure effectifs pour les magnétrons plan et cylindrique lorsque les effets des charges d'espace ne peuvent pas être négligés :

$$
\begin{aligned}
& B_{\mathrm{e}}=\sqrt{\frac{2 m}{e} \frac{(V-\varphi)^{1 / 2}}{\mathrm{~d}}} \quad \text { (plan) } \\
& B_{\mathrm{e}}=\sqrt{\frac{8 m}{e} \frac{(V-\varphi)^{1 / 2}}{R}} \quad \text { (cylindrique). }
\end{aligned}
$$

Quand on relève les caractéristiques de coupure d'un magnétron et qu'on les reporte sur un graphique $I_{\mathrm{a}} / I_{0}=f(B)$, on doit observer un déplacement du champ de coupure effectif vers les champs faibles, lorsque l'émission de la cathode augmente (courbe en pointillé sur la figure 4 correspondant à une forte émission électronique limitée par les effets de charges d'espace).

La valeur théorique du champ de coupure $B_{\mathrm{c}}$ donnée par la relation (1) doit correspondre à l'extrémité du plateau horizontal de la courbe $I_{\mathrm{a}} / I_{0}=f(B)$ pour des émissions électroniques très faibles (courbe en trait plein de la figure 4).

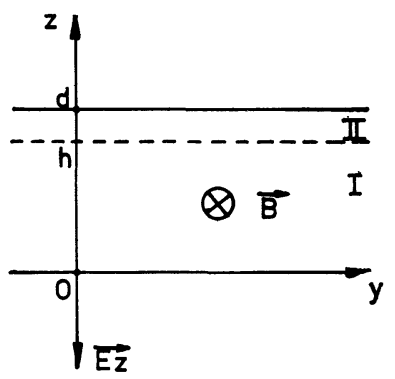

FiG. 5. - Magnétron plan muni d'une grille.

Pour obtenir une relation plus précise donnant le champ de coupure effectif $B_{\mathrm{e}}$, il faudrait pouvoir déterminer la trajectoire d'un électron individuel compte tenu de son interaction avec les électrons du nuage anodique. Ce calcul est compliqué, car il comporte la résolution d'une équation différentielle du second ordre non linéaire, et exige la connaissance (au moins approchée) de la répartition des charges d'espace entre les électrodes (cette dernière étant fonction non seulement du niveau d'émission cathodique, mais aussi du champ magnétique appliqué).

Dans le paragraphe suivant, nous analysons le cas idéal d'un magnétron plan, muni d'une grille permettant de changer à volonté la distribution du potentiel dans l'espace interélectrode.

Calcul du Ghamp de coupure efFectif $B_{\mathrm{e}}$ Dans UN MAGNÉTRON PLAN MUNI D'UNE GRILLE. - Pour des raisons de simplicité, considérons une diode à électrodes planes parallèles de dimensions infinies, le plan de la cathode étant choisi comme plan xoy, celui de l'anode ayant pour cote $z=d$, et 0 étant la position initiale d'un électron. Soit $\mathbf{B}=-B \mathbf{e}_{\mathbf{x}}$ le vecteur champ magnétique uniforme, $\mathbf{E}=-E \mathbf{e}_{\mathrm{z}}=-\frac{V}{d} \mathbf{e}_{\mathrm{z}}$ le vecteur champ électrique uniforme existant dans tout l'espace interélectrode du magnétron plan.

Les équations paramétriques de la trajectoire d'un électron quittant la cathode $\left(x_{0}=y_{0}=z_{0}=0\right)$ sont :

$$
\left\{\begin{array}{l}
y=\frac{E}{B} t-\frac{1}{\omega_{\mathrm{b}}} \frac{E}{B} \sin \omega_{\mathrm{b}} t-\frac{v_{0}}{\omega_{\mathrm{b}}} \cos \omega_{\mathrm{b}} t+\frac{v_{0}}{\omega_{\mathrm{b}}} \\
z=\frac{1}{\omega_{\mathrm{b}}} \frac{E}{B}\left(1-\cos \omega_{\mathrm{b}} t\right)+\frac{v_{0}}{\omega_{\mathrm{b}}} \sin \omega_{\mathrm{b}} t
\end{array}\right.
$$

compte tenu des hypothèses suivantes sur la vitesse initiale de l'électron $v_{\mathrm{ox}}=v_{\mathrm{oy}}=0, v_{\mathrm{oz}}=v_{\mathrm{o}} \neq 0$.

Le mouvement de cet électron (effets des charges d'espace négligés) se compose donc d'un mouvement circulaire uniforme

$$
\text { (cercle de rayon } \rho=\sqrt{\left(E / \omega_{\mathrm{b}} B\right)^{2}+v_{\mathrm{o}}^{2} / \omega_{\mathrm{b}}^{2}}
$$

et d'un mouvement rectiligne uniforme le long de l'axe oy (vitesse de dérive $W_{\mathrm{d}}=\frac{E}{B}$ ).

La valeur du champ de coupure $B_{0}$ s'obtient en écrivant que $2 \rho=d$ et en remplaçant $E$ par sa valeur en fonction de la différence de potentiel appliquée $E=\frac{V}{d}$, soit :

$$
B_{\mathrm{o}}=\sqrt{\frac{2 m}{e}} \cdot \frac{V^{1 / 2}}{d} \cdot\left(1+\frac{v_{\mathrm{o}}^{2}}{W_{\mathrm{d}}^{2}}\right)^{1 / 2}
$$

qui devient bien :

$$
B_{\mathrm{c}}=\sqrt{\frac{2 m}{e}} \cdot \frac{V^{1 / 2}}{d} \quad \text { si } \quad v_{\mathrm{o}} \ll W_{\mathrm{d}} .
$$

Si l'on avait tenu compte d'une composante de vitesse initiale $v_{\text {oy }}$, les équations paramétriques auraient été un peu plus compliquées, les résultats restant pourtant analogues; seul un terme du premier ordre en $v_{\text {oy }} / W_{\mathrm{d}}$ s'ajoute ou se retranche dans l'expression de $B_{\mathrm{c}}$ suivant le sens de $\mathbf{v}_{\mathrm{oy}}$. Dans la suite, nous supposerons que la vitesse initiale est nulle

$$
\left(v_{\mathrm{ox}}=v_{\mathrm{oy}}=v_{\mathrm{oz}}=v_{\mathrm{o}}=0\right)
$$


pour simplifier les calculs, le phénomène de déplacement du champ de coupure ne pouvant pas être expliqué par des variations possibles de celle-ci.

Considérons maintenant cette diode à électrodes planes parallèles de dimensions infinies et supposons qu'elle soit équipée d'une grille (cote $z=h<d$, cf. fig. 5) transparente aux électrons émis par la cathode. La différence de potentiel appliquée entre les électrodes étant donnée $V$, on peut à l'aide d'un deuxième générateur de tension faire varier la différence de potentiel $V_{1}$ entre la cathode et la grille. $\mathrm{Si}$ le niveau d'émission cathodique reste suffisamment faible pour que les interactions électrons-électrons entre les électrodes puissent être négligées, le magnétron plan considéré est divisé en deux régions I et II dans lesquelles champs électriques $\left(\mathbf{E}_{1}, \mathbf{E}_{2}\right)$ et magnétique (B) sont uniformes. Les calculs de la trajectoire d'un électron et du champ de coupure sont faciles à effectuer dans ce cas et le paramètre $V_{1}$ introduit, en quelque sorte, la possibilité de "simuler » l'effet des charges d'espace anodiques.

Résumons brièvement les résultats d'un tel calcul. $\mathrm{La}$ trajectoire d'un électron partant de l'origine avec une vitesse initiale nulle à l'instant $t_{0}=0$ dans la zone I est définie par les équations paramétriques I :

$$
\text { I }\left\{\begin{array}{l}
y=\frac{E_{1}}{B} t-\frac{1}{\omega_{\mathrm{b}}} \frac{E_{1}}{B} \sin \omega_{\mathrm{b}} t \\
z=\frac{1}{\omega_{\mathrm{b}}} \frac{E_{1}}{B}\left(1-\cos \omega_{\mathrm{b}} t\right) \text { avec } z \leqslant h .
\end{array}\right.
$$

Cet électron atteint le point $\mathbf{M}_{1}\left\{\begin{array}{l}y_{1} \\ z_{1}=h\end{array}\right.$ dans le plan de la grille au temps $t_{1}$ défini par la relation $z_{1}=h$; sa vitesse à cet instant est :

$$
v_{\mathrm{t}_{1}}\left\{\begin{array}{l}
\left(\frac{\mathrm{d} y}{\mathrm{~d} t}\right)_{1}=\frac{E_{1}}{B}-\frac{E_{1}}{B} \cos \omega_{\mathrm{b}} t_{1} \\
\left(\frac{\mathrm{d} z}{\mathrm{~d} t}\right)_{1}=\frac{E_{1}}{B} \sin \omega_{\mathrm{b}} t_{1} .
\end{array}\right.
$$

Les valeurs des paramètres $y_{1}, z_{1}=h,\left(\frac{\mathrm{d} y}{\mathrm{~d} t}\right)_{1}$ et $\left(\frac{\mathrm{d} z}{\mathrm{~d} t}\right)_{1}$ représentent les conditions initiales des équations du mouvement de l'électron dans la zone II.

Dans cette zone, la cote de l'électron varie comme :

II $\left\{\begin{aligned} z=-\left(\frac{1}{\omega_{\mathrm{b}}} \frac{E_{2}}{B}-h\right) \cos \omega_{\mathrm{b}} t+\frac{1}{\omega_{\mathrm{b}}} \frac{E_{2}}{B} \\ +\frac{1}{\omega_{\mathrm{b}}} \frac{E_{1}}{B} \sin \omega_{\mathrm{b}} t_{1} \sin \omega_{\mathrm{b}} t \text { avec } z \geqslant h .\end{aligned}\right.$

Nous voyons de suite que si le rayon du cercle $\rho_{1}=\frac{E_{1}}{\omega_{\mathrm{b}} B}$ qui peut servir à définir la trajectoire cycloïdale de l'électron dans la zone I est inférieur ou égal à $h / 2$, on doit tirer la condition de coupure effective à partir du système d'équations I en écrivant $2 \rho_{1}=h$; il vient :

$$
\begin{gathered}
B_{\mathrm{e}}=\sqrt{\frac{2 m}{e}} \cdot \frac{V_{1}^{1 / 2}}{h} \\
V_{1} \leqslant\left(\frac{h}{d}\right)^{2} V .
\end{gathered}
$$

avec

Par contre, si le potentiel grille $V_{1}$ est compris entre $\left(\frac{h}{d}\right)^{2} V$ et $\frac{h}{d} V$, pour obtenir la condition de coupure on doit utiliser l'équation II en écrivant $\frac{\mathrm{d} z}{\mathrm{~d} t}=0$ pour $z=d$; il vient :

$$
B_{\mathrm{o}}=\sqrt{\frac{2 m}{e}} \cdot \frac{V^{1 / 2}}{d}
$$

avec

$$
\left(\frac{h}{d}\right)^{2} V \leqslant V_{1} \leqslant\left(\frac{h}{d}\right) V \text {. }
$$

La relation (5) peut être comparée à la première équation du système (4), le changement de variable $V_{1}=(1-\alpha)\left(\frac{h}{d}\right)^{2} V$, une fois effectuée avec $0<\alpha<1$; l'expression (6) identique à celle du champ de coupure d'un magnétron plan ordinaire.

Ce raisonnement, qui nous a conduits aux relations (5) et (6), même basé sur des hypothèses qui ne correspondent pas tout à fait à la réalité, montre que le phénomène de déplacement du champ de coupure (en fonction des charges d'espace anodiques) est parfaitement plausible et ne constitue pas une entorse au principe de conservation de l'énergie.

Résultats expérimentaux. - Les résultats présentés ici concernent l'étude des caractéristiques d'un magnétron cylindrique à cathode filiforme en fonction du régime d'émission électronique. Nous ne parlerons pas ici de l'appareillage qui nous a permis de les obtenir et qui sera décrit dans un article plus spécialisé [14]. Disons simplement qu'il permet d'atteindre une pression résiduelle de $10^{-9}$ torr après étuvage et que le magnétron est constitué d'un filament de tungstène (diam. : $0,25 \mathrm{~mm}$, long. : $3 \mathrm{~cm}$ ) et d'une anode cylindrique $(R=1,35 \mathrm{~cm}$, hauteur : $2,5 \mathrm{~cm})$; un solénoïde, placé à l'extérieur de l'enceinte à vide constituée à l'emplacement du magnétron d'un tube d'acier inoxydable de diamètre $3,8 \mathrm{~cm}$, produit le champ magnétique longitudinal.

La figure 6 représente le schéma de principe du circuit électrique d'alimentation du magnétron. Le courant de chauffage et le courant magnétisant proviennent d'alimentations stabilisées en courant continu $(10 \mathrm{~V}, 10 \mathrm{~A}$ et $50 \mathrm{~V}, 10 \mathrm{~A})$. Une source de haute tension stabilisée $(0$ à $300 \mathrm{~V}, 10 \mathrm{~mA})$ et un microampèremètre R.G.A. $\left(10^{-3}\right.$ à $10^{-8} \mathrm{~A}$ par décade) servent respectivement à fournir la tension et à mesurer le 


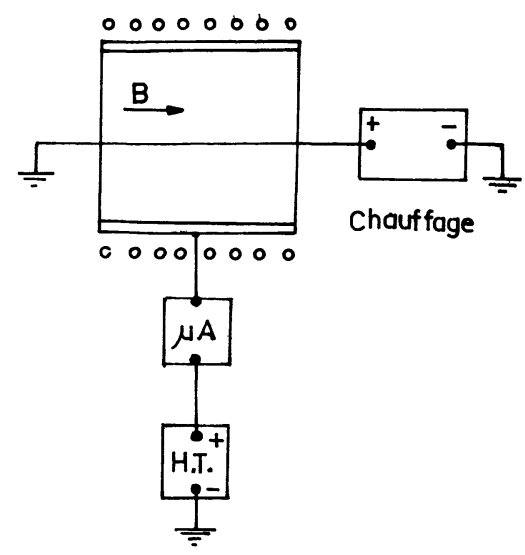

FIG. 6. - Schéma de principe du circuit électrique.

courant anodique $I_{\mathrm{a}}$. La relation donnant le champ magnétique en fonction du courant magnétisant $i_{\mathrm{S}}$ est : $B=200 i_{\mathrm{S}}$ ( $B$ gauss, $i_{\mathrm{s}}$ ampère).

L'avantage des graphiques à ordonnée sans dimension $\left(I_{\mathrm{a}} / I_{0}\right)$ réside dans le fait que l'on peut ne pas tenir compte d'effets secondaires (qui peuvent être néanmoins importants comme l'ont montré de nombreux auteurs), tels que : effets de bord, d'asymétrie (mauvais alignement du filament ou du champ magnétique), effets dus à la tension de chauffage et ceux dus au champ magnétique du courant de chauffage; en effet, toutes les courbes $I_{\mathrm{a}} / I_{0}=f(B)$, pour un potentiel d'anode donné, sont comparées à une courbe étalon correspondant par exemple à une émission importante du filament.

Le fait de pouvoir faire fonctionner le magnétron à très basse pression permet, d'autre part, de négliger

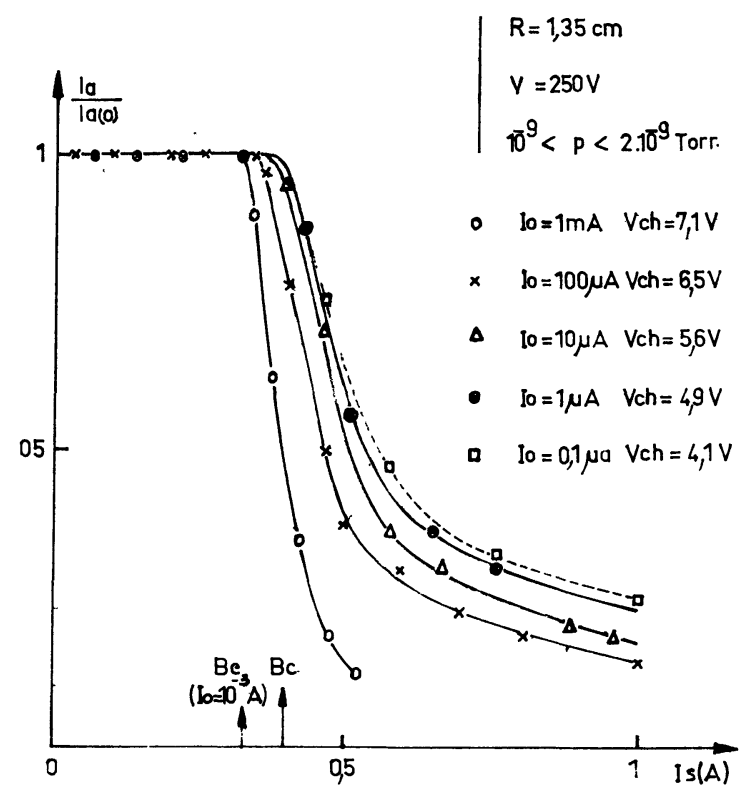

FIG. 7. - Caractéristiques expérimentales d'un magnétron cylindrique à cathode filiforme $(R=1,35 \mathrm{~cm}$, $V=250$ volts). les effets dus aux collisions d'électrons avec les molécules neutres du gaz résiduel; ces effets font l'objet d'une étude préliminaire exposée ailleurs [13].

Les caractéristiques expérimentales résumées sur la figure 7 ont été relevées point par point, en faisant varier le courant circulant dans le solénoïde. Pour obtenir un niveau d'émission électronique donné à potentiel d'anode constant ( $V_{\mathrm{a}}=250$ volts), il suffit de régler le courant de chauffage du filament. Pour chaque courant anodique $I_{2}(0)$, on a noté sur la figure 7 la différence de potentiel appliquée entre les extrémités du filament; la différence de potentiel entre l'anode et une extrémité du filament est donc exactement 250 volts, mais elle n'est que 250 volts $-V_{\text {ohauf. }}$ entre l'autre extrémité et l'anode; cet effet n'entraîne sûrement pas le déplacement observé du champ de coupure effectif puisque la variation relative de l'ordre de $1 \%$ sur $V_{\mathrm{a}}$ ne devrait provoquer qu'une variation relative de $0,5 \%$ sur $B_{0}$. Les cinq caractéristiques de coupure $\left(I_{\mathrm{a}} / I_{\mathrm{a}}(\mathrm{o})=f(B)\right.$ représentées sur la figure 7 correspondent aux cinq courants anodiques suivants : $I_{\mathrm{a}}(\mathrm{o})=10^{-3}, 10^{-4}, 10^{-5}, 10^{-6}$ et $10^{-7} \mathrm{am}-$ père; pour des courants anodiques inférieurs $\left(10^{-8}\right.$, $10^{-9}$ ampère), les courbes se confondent pratiquement avec celle correspondant à $10^{-7} \mathrm{~A}$, ce qui montre qu'à partir de ce niveau d'émission les effets de charges d'espace sont négligeables.

Dans le domaine intermédiaire $\left(10^{-6}<I_{0}<10^{-3} \mathrm{~A}\right)$, bien que le courant anodique ne soit pas limité par les charges d'espace, les effets des charges du nuage anodique deviennent de plus en plus importants (pour $B \simeq B_{\mathrm{c}}$ ) à mesure que le niveau d'émission augmente. Sur la figure 7 , une flèche indique la position du champ de coupure théorique $B_{\mathrm{c}}$ calculée à partir de la relation (1); on peut voir que cette abscisse correspond à peu près au début de la coupure (ou pente abrupte) des caractéristiques correspondant aux émissions électroniques très faibles $\left(I_{\mathrm{a}}(0) \leqslant 10^{-6} \mathrm{~A}\right)$. Le champ de coupure effectif $B_{\theta}$ correspondant à un courant anodique $I_{\mathrm{a}}(\mathrm{o})=10^{-3} \mathrm{~A}$, lui, est inférieur à cette valeur $B_{\mathrm{c}}$ d'environ $12 \%$.

Notons tout de suite qu'un effet analogue est observable sur les caractéristiques publiées par Hull dans le domaine des fortes émissions. La figure 8 représente les résultats de Hull (fig. 12 de la référence [1]) obtenus avec un magnétron cylindrique à cathode filiforme $\left(R=1,27 \mathrm{~cm}, V_{\mathrm{a}}=250\right.$ volts) transcrits sur un graphique $I_{\mathrm{a}} / I_{\mathrm{a}}(\mathrm{o})=f(B)$. La figure 8 indique nettement un déplacement du champ de coupure effectif vers les champs faibles lorsque l'émission augmente.

Enfin, dans le domaine des émissions très faibles et des pressions résiduelles de l'ordre de $10^{-9}$ torr, Lafferty a effectué des relevés de caractéristiques de coupure à l'aide d'une diode cylindrique de dimensions semblables mais avec un filament en forme d'épingle à cheveux sur l'axe de l'anode.

Sur la figure 9 , on a représenté deux courbes $\alpha$ et $\gamma$ tirées des figures 3 et 7 de son article [9]. La courbe $\alpha$ 


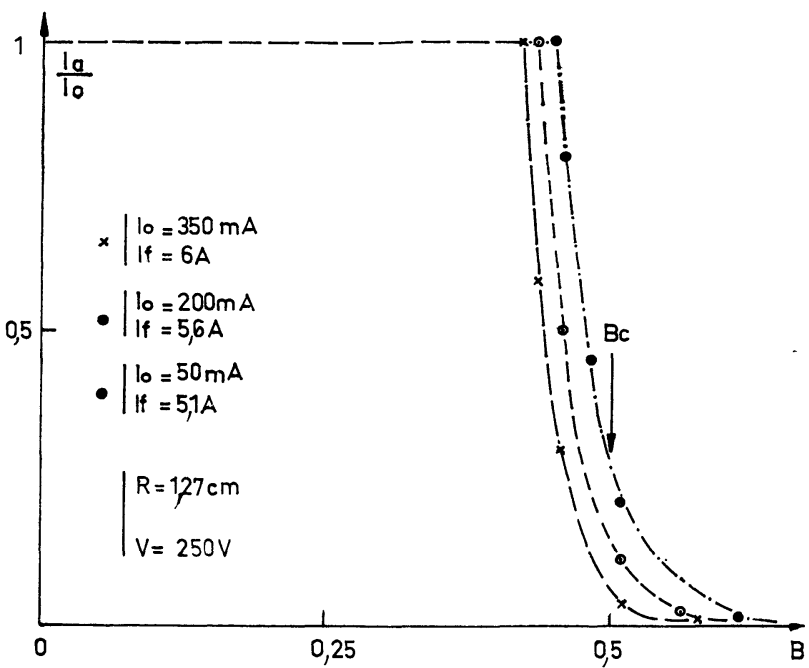

FIG. 8. - Résultats de Hull

$(R=1,27 \mathrm{~cm}, V=250$ volts $)$.

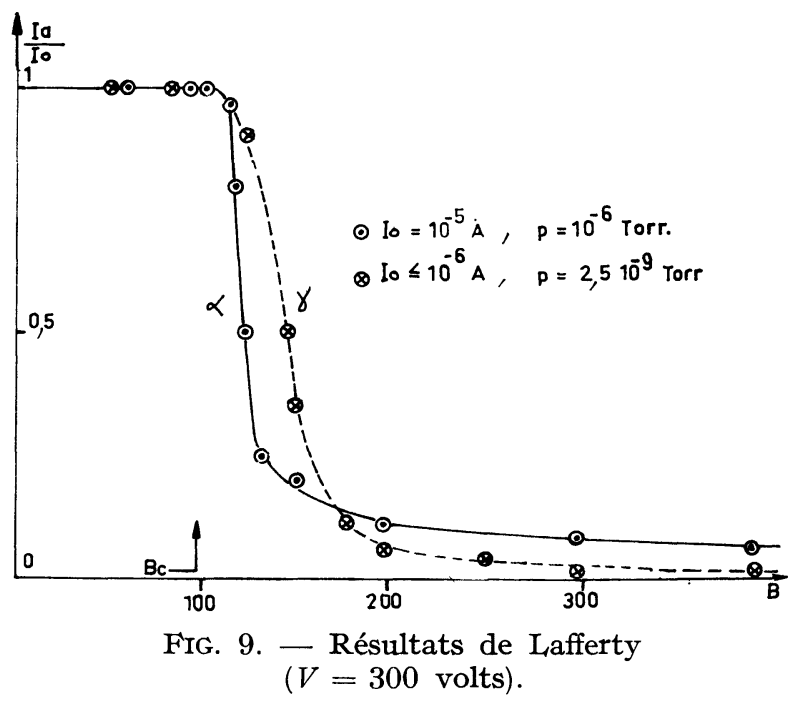

correspondant à $I_{\mathrm{a}}(\mathrm{o})=10^{-5} \mathrm{~A}$ sert de référence : le fait que le champ de coupure mesuré soit nettement supérieur au champ de coupure théorique $B_{\mathrm{c}}$ peut s'expliquer par la forme en épingle à cheveux du filament utilisé. La courbe $\gamma$, correspondant à des émissions électroniques très faibles $\left(I_{0} \leqslant 10^{-6} \mathrm{~A}\right)$, est déplacée par rapport à la précédente $\left(I_{0}=10^{-5} \mathrm{~A}\right)$ vers les champs croissants : les pressions résiduelles correspondant aux deux cas $\alpha$ et $\gamma$ sont respectivement $10^{-6}$ et $2,5 \times 10^{-9}$ torr. On peut remarquer sur la figure 9 (ainsi d'ailleurs que sur la figure 7) que le blocage de la diode se produit plus rapidement pour une émission électronique plus importante, même lorsque les effets des collisions électrons-molécules neutres ne sont pas tout à fait négligeables (courbe $\alpha$ reste au-dessus de $\gamma$ pour $B>200$ gauss). En effet, la transition entre le régime de débit et le régime de blocage est plus rapide pour des émissions électroniques élevées que pour les émissions électroniques très faibles : ce phénomène peut d'ailleurs s'expliquer par l'influence plus importante de la distribution des vitesses initiales non nulles (approximativement maxwellienne suivant Nottingham [12]) dans le cas des faibles émissions.

Conclusion. - En conclusion, nous avons montré que la condition de coupure d'un magnétron dépend du niveau d'émission électronique de la cathode, que la valeur théorique du champ de coupure

$$
B_{0}=\sqrt{\frac{8 m}{e}} \cdot \frac{V^{1 / 2}}{R}
$$

d'un magnétron cylindrique à cathode filiforme doit correspondre sur les caractéristiques $I_{\mathrm{a}} / I_{\mathrm{o}}=f(B)$ à l'extrémité du plateau horizontal lorsque l'émission cathodique est très faible (effets des charges d'espace négligés). Dès que les effets des charges d'espace ne peuvent plus être négligés, un déplacement du champ de coupure vers les champs faibles est observé; en d'autres termes, le champ magnétique entraîne la formation d'un nuage électronique au voisinage de l'anode qui aide à une coupure plus efficace et plus rapide du courant anodique. Ces conclusions sont confirmées par nos résultats expérimentaux, ainsi d'ailleurs que par des résultats publiés antérieurement [1], [9] pour des magnétrons cylindriques de dimensions analogues.

Ce travail a pu être effectué grâce à un contrat de recherches accordé par la Direction des Recherches et Moyens d'Essais (D.R.M.E.).

Manuscrit reçu le 15 avril 1967.

\section{BIBLIOGRAPHIE}

[1] Hull (A. W.), Phys. Review, 1921, 18, 31.

[2] Briliouin (L.), J. Physique Rad., 1940, 1, 233.

[3] AlLIs (W. P.), M.I.T. Radiation Lab. Report, 1942, 3, 43.

[4] Page (L.) et Adams (N. I.), Phys. Review, 1946, 69, 492.

[5] Delcroix (J.-L.), Thèse, Paris, 1954, Étude des propriétés statiques des charges d'espace du type magnétron.

[6] CosTe (J.), Thèse, Paris, 1961, Magnétron à anode lisse. Théorie statistique et oscillations ordonnées.
[7] ReVerdin (J. L.), Journal Applied Phys., 1951, 22, 257.

[8] REDHEAd (P. A.), Can. Journal Phys., 1959, 37, 1260.

[9] LAFFERTY (J. M.), Journal Applied Phys., 1961, 32, 424.

[10] Kaufman (H. R.), N.A.S.A., T.N.D., 1961, 585.

[11] LANGMUIR (I.), Phys. Review, 1913, 2, 455.

[12] Notitingham (W. B.), Handbuch der Physik, 1956.

[13] ROCARD (J.-M.) et LABORIE (P.), Rapport R.D.M.E., L.P.T., 6, oct. 1966.

[14] Capdevilita (A.), Thèse de Doctorat de spécialité (à publier), Toulouse. 